\title{
La protección jurídica ambiental del agua para consumo humano. Panorama de dos provincias argentinas y del ámbito internacional ${ }^{1}$
}

\section{The environmental legal protection of water for human consumption. Overview of two argentine provinces and the international sphere}

Recibido: 05 de julio de 2015 - Revisado: 21 de enero de 2016 - Aceptado: 12 de abril de 2016

Clara María Minaverry ${ }^{2}$

\section{Resumen}

El objetivo del presente artículo es analizar el nivel de evolución de la protección jurídica ambiental del agua para consumo humano en la normativa de las provincias de Buenos Aires y Santa Fe (Argentina) y en el ámbito internacional, a través de la aplicación de tres de los paradigmas de desarrollo elaborados por Michael Colby: a) protección ambiental, b) administración de los recursos y c) ecodesarrollo. La metodología utilizada es cualitativa, fundamentalmente la hermenéutica jurídica. Podemos concluir que ambos ordenamientos jurídicos provinciales poseen un fuerte enfoque antropocéntrico, al privilegiar los intereses del hombre por sobre los derechos de la naturaleza/ambiente, y no profundizan en aspectos esenciales como son los sociales, ambientales y culturales.

\section{Palabras clave}

Derecho ambiental, agua, normas jurídicas, Argentina, internacional.

\begin{abstract}
The objective of this article is to analyze the level of evolution of environmental legal protection of water for human consumption in the regulations of the provinces of Buenos Aires and Santa Fe (Argentina) and in the international sphere, through the application of three of the development paradigms worked out by Michael Colby: a) environmental protection, b) resource management and c) eco development. The methodology used is qualitative, fundamentally legal hermeneutics. We can conclude that both provincial legal systems have a strong anthropocentric approach, privileging the interests of man over the rights of nature/environment, and do not delve into essential aspects such as social, environmental and cultural.
\end{abstract}

\section{Keywords}

Environmental law, water, legal norms, Argentina, international.

\footnotetext{
${ }^{1}$ Este artículo se vincula con el proyecto de investigación titulado "La inevitable relación entre la protección de los recursos hídricos y de los bosques. Situación legal en Argentina y análisis comparativo con Uruguay" Dirigido por la Dra. Clara María Minaverry, Universidad Nacional de Luján, Argentina.

2 Abogada de la Universidad de Buenos Aires, Argentina. Magister en Derecho Ambiental de la Universidad Complutense de Madrid, España y Doctora en Derecho de la Universidad de Buenos Aires. Investigadora del Consejo Nacional de Investigaciones Científicas y Técnicas (CONICET) y del Instituto de investigaciones Ambrosio Gioja, Facultad de Derecho, Universidad de Buenos Aires. Profesora Adjunta Ordinaria de Derecho Ambiental de la Universidad Nacional de Luján y de posgrado de la Universidad de Buenos Aires y de la Universidad Tecnológica Nacional, correo electrónico: cminaverry@derecho.uba.ar

Para citar este artículo use: Minaverry, C. (2016). La protección jurídica ambiental del agua para consumo humano. Panorama de dos provincias argentinas y del ámbito internacional. Civilizar Ciencias Sociales y Humanas, 16(31), 17-40.
} 


\section{Introducción}

La problemática del agua ya no es un asunto aislado, sin atención en la actualidad y ocupa la agenda de la mayoría de los países del mundo. La escasez del agua dulce, ocasionada por el aumento de la población que demanda y la contaminación por las actividades antrópicas, lleva a países a declarar el líquido vital como bien estratégico y formular hipótesis de guerras futuras por su posesión (De Bianchetti, 2010, p. 1).

Esto tiene su razón de ser fundamentalmente porque la crisis ambiental es contemporánea, no es ecológica sino social y se vincula con nuestra actual civilización (Galano et al, 2002, p. 1). Por una ética para la sustentabilidad" en 2002. Además, se puede relacionar con parámetros de "valoración" que se visualizan dentro de un enfoque individualista u holístico. En este último caso se incluyen a dos o más individuos, quienes se conectan de modo arbitrario o al azar con otras especies genética o ecosistémicamente si se trata de territorios (Curry, 2006, p. 40).

Coincidimos en que "valorar no siempre significa atribuir un valor monetario" (Martínez -Alier, 2015, p. 67) y más tratándose de cuestiones que se asocian con la protección del ambiente, donde se debe tener una multiplicidad de factores. Cuando hacemos referencia al agua para consumo humano apuntamos a que la misma posea una buena calidad (medida a través de la utilización de tecnología), según lo que establecen las organizaciones internacionales y nuestra propia legislación, para que no se genere ningún daño a la salud humana.

No hay que olvidar que los medios naturales absorben diferentes contaminantes por la vía de emisiones de material particulado, vertimientos, metales pesados, agroquímicos, entre otros, cambiando sus propiedades como con la eutrofización de los medios acuáticos (Guzmán-Aguilera, 2013, p. 78). Es fundamental establecer que
Dos cosas interesan del agua: la cantidad y la calidad. La cantidad alude al agua disponible; para lo cual es necesario realizar estadísticas, hacer inventarios sobre el estado del agua dulce, del agua potable, etc. La calidad en cambio, está más asociada al uso que se le pretende dar: para beber, para riego, o sea el tipo de agua que se requiere, según los usos a los que se la destine (De Bianchetti, s.f., p. 3).

Aproximadamente desde la década del ochenta, el derecho ambiental internacional tiene un papel trascendental, pues suministra instrumentos que contienen principios respecto de los cuales algunos Estados los han venido receptando e incorporando en su normativa interna (como es el caso de Argentina).

Las primeras herramientas internacionales fueron la Declaración de Estocolmo de 1972, la Carta de la Naturaleza de 1982 y la Carta de la Tierra del año 2000, que sumaron temáticas vinculadas con la conservación de la naturaleza, la integridad ecológica y la paz, entre otros asuntos.

Además en América Latina la corriente del "constitucionalismo latinoamericano", fue destacada por diversos autores que establecen que en la misma se produjo la recepción de los convenios internacionales de derechos humanos, la búsqueda de los criterios de interpretación más favorables para las personas o las acciones directas de amparo, y que otorgan a los derechos sociales, ampliamente reconocidos, la máxima efectividad (Viciano-Pastor \& Martínez-Dalmau, s.f., p. 21).

Esto plantea importantes desafíos jurídicos, donde se debe procurar llevar exitosamente a la práctica postulados tales como el reconocimiento de la naturaleza como sujeto de derechos (como ocurrió en la Constitución de Ecuador en 2008). A su vez, la región se encuentra inserta en un contexto asociado con las premisas de la moderna teoría de la política verde, que toma en consideración a la evolución permanente del derecho, de la institucionalidad 
y de las políticas públicas, hacia un modelo de Estado centrado en las preocupaciones ecológicas (Molina-Roa, 2014, p. 153). Esto se enlaza directamente con que

[...] el aire o el agua, son recursos que trascienden las fronteras, motivo por el cual los Estados deben coordinar sus políticas en vistas a la preservación de tan esencial recurso. Este recurso hídrico puede a su vez ir asociado con grandes problemas climáticos: sequías o su antítesis: las inundaciones (De Bianchetti, s.f., p. 1).

En Argentina, antes de la reforma constitucional de 1994, el Congreso Nacional solo podía legislar mediante los códigos de fondo y en las materias y casos que la Constitución Nacional le había delegado. Todo lo ambiental ajeno a esos códigos y materias competía a los poderes locales, y cada provincia argentina poseía la exclusiva potestad de gestionar lo relativo con el ambiente y los recursos naturales.

El artículo 41 del texto constitucional reformado en 1994 facultó al Congreso Nacional para dictar leyes de presupuestos mínimos ambientales (Valls, 2012, p. 7), "y a las provincias, las necesarias para complementarlas". Se definieron en el artículo 6 de la Ley General del Ambiente (25.675) de la siguiente manera:

Se entiende por presupuesto mínimo, establecido en el artículo 41 de la Constitución Nacional, a toda norma que concede una tutela ambiental uniforme o común para todo el territorio nacional, y tiene por objeto imponer condiciones necesarias para asegurar la protección ambiental. En su contenido, debe prever las condiciones necesarias para garantizar la dinámica de los sistemas ecológicos, mantener su capacidad de carga y, en general, asegurar la preservación ambiental y el desarrollo sustentable.

Este relativamente nuevo concepto implica mayor compromiso normativo por parte de las provincias, lo cual muchas veces resulta ser muy controversial, ya que las mismas afirman que ellas son titulares del derecho a gestionar sus recursos naturales, amparándose en el derecho de propiedad (Constitución Nacional, art. 17) y en el artículo 124 de la Constitución Nacional, que establece en su último párrafo que "corresponde a las provincias el dominio originario de los recursos naturales existentes en su territorio".

De acuerdo con el contexto jurídico descripto, el objetivo del presente artículo es analizar el nivel de evolución de la protección jurídica ambiental del agua para consumo humano en la normativa de las provincias de Buenos Aires y Santa Fe, en Argentina, y en el ámbito internacional, a través de la aplicación de tres de los paradigmas de desarrollo elaborados por Michael Colby: a) protección ambiental, b) administración de los recursos y c) ecodesarrollo.

Primero, el paradigma de protección ambiental en lugar de centrarse en los procedimientos para mejorar las acciones de desarrollo y la resistencia ecológica, tiene una postura defensiva o correctiva en la práctica, y se lo describe como el enfoque del "final del tubo" o de "lo mismo de siempre, más una planta de tratamiento" (Colby, 1998, p. 599). El mismo se traduce en la intención de mantener el statu quo respecto de los actuales niveles de protección del ambiente.

Segundo, el paradigma de la administración de los recursos,

[...] incluye a todos los tipos de capital y de recursos -biofísicos, humanos, infraestructurales y monetarios- en los cálculos de las cuentas nacionales, la productividad y las políticas de desarrollo y de planeamiento de la inversión. El clima y los procesos que lo regulan se están considerando ahora como un recurso fundamental y vital, que debe administrarse según este paradigma (Colby, 1998, p. 602.

El mismo se vincula con las formas o estrategias de organización que requiere el Estado para que la sociedad pueda beneficiarse de todo lo que brinda la naturaleza, y debe administrarse económicamente de modo adecuado. 
Tercero, el ecodesarrollo intenta reestructurar la relación existente entre la sociedad y la naturaleza en un "juego de suma positiva", mediante la reorganización de las actividades humanas para que sean sinérgicas con los procesos y los servicios de los ecosistemas, por oposición a la "simbiosis sencilla" de regreso a la naturaleza defendida por los ecologistas profundos (Colby, 1998, p. 604).

En este último paradigma se traza el propósito de realizar modificaciones sustanciales en la realidad, con objetivos más trascendentales $\mathrm{y}$ de forma inmediata, y muchas veces es denominado por algunos autores como el enfoque "ecosistémico", en donde se intenta mantener un equilibrio entre las actividades humanas, las condiciones y los beneficios que brinda la naturaleza. Se plantea la necesidad de realizarse una reforma o modificación del statu quo de la reglamentación vigente, para que directa o indirectamente a través de la elaboración de algún programa, plan, proyecto o norma jurídica se pueda colaborar para lograr tales fines.

En este sentido, coincidimos con algunos autores que afirman que el derecho ambiental debería funcionar en conjunto con los conceptos que brindan otras ciencias, como lo es la ecología política, que no tiene como objetivo analizar los conflictos socioambientales sino solucionar problemas tales como la pérdida de biodiversidad, el cambio climático o las contaminaciones locales (Martínez-Alier, 2015, p. 58).

La ecología política al igual que el poder político, incide en los conflictos socioambientales (Martínez-Alier, 2015, p. 57), y aquí se debería incluir el derecho ambiental a través del diseño y elaboración de diversas políticas públicas, ya que en la práctica y en la mayoría de los casos, lo ambiental y lo político son tratados de forma aislada e independiente entre sí, en el sentido de que no cruzan sus conocimientos. Esto sin duda genera un impacto negativo en el nivel de desarrollo normativo ambiental, separándolo de la realidad que excede lo estrictamente jurídico, y por lo tanto limitando la extensión de su protección.

\section{Metodología}

Se adoptó un diseño de tipo bibliográficodocumental, descriptivo y analítico comparativo entre las normas jurídicas de las provincias de Buenos Aires y Santa Fe (ambas en Argentina) y los instrumentos internacionales sobre protección ambiental y del agua. La metodología que se utilizó es cualitativa, fundamentalmente la hermenéutica jurídica.

Se recurrió a diversas fuentes y técnicas de recolección de datos, y se consultaron estudios académicos, fuentes judiciales y materiales oficiales y públicos (pertenecientes al ámbito nacional y provincial de Argentina y al internacional). El trabajo se dividió en tres etapas:

1. Fase exploratoria: en un primer momento se recopiló una selección de normas jurídicas y de doctrina vinculada con la protección ambiental y con la calidad del agua potable de las jurisdicciones de Buenos Aires y Santa Fe y del ámbito internacional. A tal fin se emplearon buscadores jurídicos y se consultaron bibliotecas de universidades y de institutos de investigación.

2. Fase descriptiva: la información recogida en la etapa anterior se clasificó y analizó, y se fueron determinando los lineamientos cardinales que surgían de las normas jurídicas y de los instrumentos internacionales, se incorporaron y clasificaron dentro de cuadros de triple entrada de acuerdo con los postulados establecidos en tres de los cinco paradigmas de desarrollo de Colby: protección ambiental, administración de los recursos y ecodesarrollo.

3. Fase analítica: con base en la instrumentalización de la clasificación de las normas jurídicas y herramientas internacionales dentro de los cuadros mencionados en la etapa precedente y luego de su descripción analítica, se desarrollaron recomendaciones y conclusiones. 
El ámbito territorial seleccionado se justifica en la necesidad de contrastar dos provincias argentinas con un relevante desarrollo normativo sobre protección ambiental y del agua, y en tener en cuenta el derecho ambiental internacional. El ámbito temporal se cimienta en que desde 1972 a la fecha, a partir de la Declaración de Estocolmo, el mundo comenzó a tomar más conciencia sobre la importancia del cuidado del ambiente, que trasladó a sus agendas políticas y a su normativa interna.

\section{La protección normativa del agua en la provincia de Buenos Aires}

A los fines de poder acceder a una adecuada calidad del agua requerida para el consu- mo humano, deben tenerse en cuenta los aportes de otras ramas del derecho y de otras disciplinas relacionadas, como son las ingenierías, la biología, la sociología, la antropología, la economía, entre otras.

Además, la finalidad que persigue el derecho ambiental resulta ser clave como disciplina en sí misma, y su mera existencia explica en muchos aspectos la obligación legal de protección de la calidad del servicio, de la población, de los recursos naturales y de los ecosistemas involucrados. A continuación se describirá la principal normativa de la provincia de Buenos Aires, y se clasificarán algunos artículos de la misma conforme con los criterios establecidos por tres de los paradigmas de desarrollo de Michael Colby.

Tabla 1

Normativa vinculada con la protección ambiental del agua para consumo humano, provincia de Buenos Aires

\begin{tabular}{|cccc|}
\hline Fuente legal & $\begin{array}{c}\text { Paradigma protección } \\
\text { ambiental }\end{array}$ & $\begin{array}{c}\text { Paradigma administración } \\
\text { de los recursos }\end{array}$ & $\begin{array}{c}\text { Paradigma } \\
\text { ecodesarrollo }\end{array}$ \\
\hline $\begin{array}{c}\text { Constitución de la Provincia de } \\
\text { Buenos Aires }\end{array}$ & Art. 28 & Art. 28 & Art. 28 \\
\hline $\begin{array}{c}\text { Decreto } 878 \text { de } 2003 \text {, provincia } \\
\text { de Buenos Aires }\end{array}$ & Art.6 inciso fy art. 24 & Arts. 24 y 26 & Arts. 93 y siguientes \\
\hline Ley $12.257^{2}$ & Art. 25 & Art. 25 & - \\
\hline Ley $14.782^{3}$ & Arts. 3 y 5 & Arts. 3 y 5 &
\end{tabular}

Fuente: elaboración propia.

La Constitución de la Provincia de Buenos Aires únicamente hace referencia a la promoción de acciones que eviten la contaminación del agua, y a asegurar políticas de conservación y de recuperación de su calidad. Sin embargo, en ella no se brinda ninguna herramienta jurídica para poder lograr dicha protección, ni tampoco se instó al Gobierno provincial para que las dicte.

El artículo 28 de esta Carta Magna puede incorporarse sin lugar a dudas en los tres paradigmas de desarrollo de Michael Colby, teniendo en cuenta algunas salvedades al texto que se describen a continuación:

Los habitantes de la Provincia tienen el derecho a gozar de un ambiente sano y el deber de conservarlo y protegerlo en su provecho y en el de las generaciones futuras.

La Provincia ejerce el dominio eminente sobre el ambiente y los recursos naturales de su territorio incluyendo el subsuelo y el espacio aéreo correspondiente, el mar territorial y su lecho, la plataforma continental y los recursos 
naturales de la zona económica exclusiva, con el fin de asegurar una gestión ambientalmente adecuada.

En materia ecológica, deberá preservar, recuperar y conservar los recursos naturales, renovables y no renovables del territorio de la Provincia; planificar el aprovechamiento racional de los mismos; controlar el impacto ambiental de todas las actividades que perjudiquen al ecosistema; promover acciones que eviten la contaminación del aire, agua y suelo; prohibir el ingreso en el territorio de residuos tóxicos o radiactivos; y garantizar el derecho a solicitar y recibir la adecuada información y a participar en la defensa del ambiente, de los recursos naturales y culturales.

Asimismo, asegurará políticas de conservación y recuperación de la calidad del agua, aire y suelo compatible con la exigencia de mantener su integridad física y su capacidad productiva, y el resguardo de áreas de importancia ecológica, de la flora y la fauna.

Toda persona física o jurídica cuya acción u omisión pueda degradar el ambiente está obligada a tomar todas las precauciones para evitarlo.

En particular cuando se alude al ámbito ecológico, podemos destacar una incipiente orientación hacia los postulados esgrimidos por el enfoque del paradigma del ecodesarrollo, al hacerse referencia a preservar los recursos naturales, y al controlar actividades que perjudiquen el ecosistema, por encima de los intereses humanos.

Luego, los paradigmas de protección ambiental y de administración de los recursos son visualizados fácilmente aquí, también al mencionarse la implementación de políticas, medidas de precaución y a través de la mención del principio de desarrollo sostenible. Uno de los objetivos centrales del decreto 878 de $2003^{4}$ es el de proteger a la salud pública, los recursos hídricos y al medioambiente (artículo 6, inciso f). En su artículo 24 regula las condiciones de la prestación así:

El servicio público sanitario deberá prestarse en condiciones que garanticen su continuidad, regularidad, cantidad, calidad y universalidad, asegurando una prestación eficaz a los usuarios y la protección de la salud pública y el medio ambiente, según las pautas que se correspondan con el servicio sustentable.

La sustentabilidad (en este caso) implica que el recurso del agua deberá estar al servicio de los intereses del hombre, manifestando de esta manera una fuerte visión antropocéntrica. El artículo 26 determina textualmente lo siguiente:

Se considera que la prestación de un servicio es sustentable cuando se logra equilibrar la oferta y demanda del mismo, donde la cobertura, calidad, inversiones, productividad y tarifas que reciben los usuarios en el presente y en el futuro responden a un equilibrio, constituyendo la tarifa el principal elemento a tener en cuenta $[\ldots]$.

El mismo hace directa referencia al paradigma de la administración de los recursos, considerando que la sostenibilidad se cumple únicamente con el aspecto económico, y sin tener en cuenta los aspectos sociales y ambientales. El Código de Aguas, ley 12.257 de 1999, es la normativa más relevante sobre este tema en la provincia de Buenos Aires, y desarrolla adecuadamente el principio central sobre gestión integral de recursos naturales (Pastorino, 2014, p. 262). En este sentido el artículo 25 también se vincula directamente con la presente temática:

Toda persona podrá usar el agua pública a título gratuito y conforme a los reglamentos generales, para satisfacer necesidades domésticas de bebida e higiene, transporte gratuito de personas o cosas, pesca deportiva y esparcimiento sin ingresar en inmueble ajeno. No deberá contaminar el medio ambiente ni perjudicar igual derecho de terceros. 
Aquí se reconoce el derecho básico de cualquier persona de poder satisfacer sus necesidades teniendo acceso al agua, pero sin descuidar la protección del ambiente (se otorgan derechos pero se solicitan contraprestaciones al usuario). Los otros artículos del Código atañen a los procedimientos y a los derechos de uso del agua de los propietarios y de concesionarios, entre otros. Sin embargo, no se han incorporado en el mismo los aspectos relativos a la protección del ambiente y del recurso dirigidos en particular hacia la protección del agua potable, a pesar de haberlo hecho en relación con el agua atmosférica.

Una de las principales ventajas del Código de Aguas es que sistematiza sus artículos en un único instrumento, lo cual facilita su aplicación en la práctica. Por el contrario, la realidad jurídica argentina indica que la mayor parte de la normativa sobre aguas se encuentra incorporada al derecho de fondo (como es el caso del Código Civil o normas administrativas) y es dispersa. La principal dificultad que tuvo dicho cuerpo normativo fue que recién se reglamentó en el año 2007 mediante el decreto 3511 del mismo año. En este sentido el artículo 25 del decreto reglamentario dicta:

El aprovechamiento de agua para satisfacer necesidades domésticas de bebida e higiene en áreas alcanzadas por el servicio público de provisión de agua potable deberá efectuarse en las condiciones que establezca la regulación de dicho servicio público, establecida en el decreto $878 / 03$ y sus normas modificatorias y complementarias.

Luego en los artículos 93 y siguientes se consigna que "el Código prevé la necesidad de evaluaciones de impacto y auditorías para realizar obras, siendo la autoridad del agua quien determinará en qué caso corresponde" (Pastorino, 2014, p. 32), y se podría considerar que estos conceptos pertenecen al ámbito del enfoque del ecodesarrollo.

La herramienta de la evaluación de impacto, en especial si se profundiza en el área social, provee la posibilidad de brindarnos el espacio para que se analicen diversos elementos que no son habitualmente regulados por la normativa sobre aguas, y así poder intentar lograr algún aporte o modificación tendiente a una protección de carácter más integral del ambiente y no tan sectorizada.

La ley 13.230 de 2004 que adhiere al Código Alimentario Nacional en su artículo 982 menciona las condiciones que debe reunir el agua para poder ser consumida por los seres humanos:

Con las denominaciones de agua potable de suministro público y agua potable de uso domiciliario, se entiende que la que es apta para la alimentación y el uso doméstico: no deberá contener substancias o cuerpos extraños de origen biológico, orgánico, inorgánico o radiactivo en tenores tales que la hagan peligrosa para la salud. Deberá presentar sabor agradable y ser prácticamente incolora, inodora, límpida y transparente. El agua potable de uso domiciliario es el agua proveniente de un suministro público, de un pozo o de otra fuente, ubicada en los reservorios o depósitos domiciliarios. Ambas deberán cumplir con las características físicas, químicas y microbiológicas $[\ldots]$.

Lo relevante es lo que se determinó en la segunda parte del texto del artículo:

[...] la autoridad sanitaria competente podrá admitir valores distintos si la composición normal del agua de la zona y la imposibilidad de aplicar tecnologías de corrección lo hicieran necesario. Para aquellas regiones del país con suelos de alto contenido de arsénico, se establece un plazo de hasta 5 años para adecuarse al valor de $0,01 \mathrm{mg} / \mathrm{l}$. (Modificado por Resolución Conjunta SPReI N $\mathrm{N}^{\circ} 34 / 2012$ y SAGyP $N^{\circ}$ 50/2012): Prorrógase el plazo de cinco (5) años previsto para alcanzar el valor de $0,01 \mathrm{mg} / \mathrm{l}$ de arsénico hasta contar con los resultados del estudio "Hidroarsenicismo y Saneamiento Básico en la República Argentina - Estudios básicos para el establecimiento de criterios y prioridades sanitarias 
en cobertura y calidad de aguas" cuyos términos fueron elaborados por la Subsecretaría de Recursos Hídricos del Ministerio de Planificación Federal.

Estos valores se vienen prorrogando desde hace algunos años, y como consecuencia, los jueces dictan precedentes jurisprudenciales con el objeto de subsanar esta situación, en donde en su mayoría se le solicita a las empresas que suministran el servicio de agua, que se adecúen a lo exigido por la Organización Mundial de la Salud, en tanto que los niveles de arsénico deberán encontrarse en el valor de $0,01 \mathrm{mg} / 1$ (Minaverry \& Cáceres, 2016, p. 73).

La última norma 14.782 que fue sancionada en la provincia de Buenos Aires data de fines del año 2015, y reconoce el acceso al agua potable y al saneamiento como un derecho humano esencial para la vida. Uno de los aspectos más interesantes y que se considera un avance normativo en la presente temática, hace referencia a que se haya regulado sobre este punto en especial, pero además que se haya definido claramente qué es lo que se entiende por este derecho humano.

En tal sentido, se estableció que se trata del "derecho de todas las personas a disponer oportunamente de agua suficiente, salubre, aceptable y accesible para el consumo humano y el uso personal y doméstico" (ley 14.782 de 2015, art. 2.1). Ninguno de estos adjetivos se explicó en el texto normativo, lo que dificulta su aplicación práctica y concreta en la realidad, sobre todo teniendo en cuenta las desfavorables condiciones de acceso al servicio público del agua en dicha jurisdicción ${ }^{5}, \mathrm{y}$ que aún dicha norma no fue reglamentada. En específico en su artículo 3, la ley hace referencia a cuales acciones puntuales se traduce este concepto:

a) El acceso oportuno a la cantidad de agua que sea necesaria y apta para el con- sumo y el uso personal y doméstico, y para promover la salud pública.

b) El acceso físico a instalaciones o servicios de agua que proporcionen el suministro necesario y regular de agua salubre.

c) La distribución equitativa y no discriminatoria de todas las instalaciones y servicios de agua potable disponibles.

d) La adopción de estrategias y planes de acción provincial sobre el agua para toda la población, que deberán ser elaborados y revisados periódicamente con base en un proceso participativo y transparente.

e) La vigilancia sobre el grado de realización del derecho al agua y al saneamiento. f) La puesta en marcha de programas de acceso al agua y al saneamiento destinados a los grupos vulnerables.

g) La adopción de medidas adecuadas para prevenir, tratar y controlar las enfermedades asociadas al agua.

h) Establecer un nivel mínimo esencial de disponibilidad diaria de agua potable por persona, que permita cubrir las necesidades básicas de consumo y para el uso personal y doméstico, y garantizarán su pleno acceso.

A su vez, más allá de tratarse de una norma específica sobre agua, en uno de sus artículos (el 5to), se alude a un aspecto relacionado con la protección ambiental, ya que se menciona la obligación para la autoridad de aplicación de la ley de tener que utilizar tecnologías económica, social y ambientalmente apropiadas. En la misma no se detalla cuáles son consideradas con estas características, pero se podría interpretar que son las que menos impactan negativamente en el ambiente, y que muchas veces resultan de difícil acceso para algunos países debido a que requieren de inversiones muy costosas.

Aunque la temática de la norma es relevante como lo es el reconocimiento de un derecho humano en el ámbito interno de un país, 
los textos hacen referencia a lo que establecen los paradigmas de protección ambiental y la administración de los recursos, ya que regulan aspectos correctivos y defensivos en relación con el agua para consumo humano, y no se innova al intentar reestructurar la relación existente entre el agua, la sociedad y el Estado.

\section{La protección normativa del agua en la provincia de Santa Fe}

A continuación se destaca la principal normativa correspondiente a la provincia de Santa $\mathrm{Fe}$, en relación con la protección ambiental del agua para consumo humano.

Tabla 2

Normativa vinculada a la protección ambiental del agua para consumo humano, provincia de Santa Fe

\begin{tabular}{|cccc}
\hline Fuente legal & $\begin{array}{c}\text { Enfoque protección } \\
\text { ambiental }\end{array}$ & $\begin{array}{c}\text { Enfoqueadministración } \\
\text { de los recursos }\end{array}$ & $\begin{array}{c}\text { Enfoque } \\
\text { ecodesarrollo }\end{array}$ \\
\hline Constitución de la Provincia de Santa Fe & - & Arts. 1 y 2 & - \\
\hline Ley 11.220 de $1994^{8}$ & Arts. 1 y 2 & Arts. 1 y 2 & - \\
\hline Ley 13.036 de $2009^{9}$ & Arts. 1 y 2 & -
\end{tabular}

Fuente: elaboración propia.

La ley 11.220 sobre transformación del sector público de agua potable, desagües cloacales y saneamiento dispone la regulación de la prestación del servicio del agua, y prevé los sistemas para la autorización de la provisión por los prestadores en todo el ámbito de la provincia de Santa Fe.

Tiene como objetivo la protección contra la contaminación del ambiente y de los recursos naturales, en especial al intentar evitar la contaminación hídrica. Esto tiene sentido, porque los contratos de concesión del servicio se han incorporado cláusulas destinadas a la protección ambiental que no son suficientemente efectivas en la práctica, faltando herramientas y mecanismos jurídicos para su aplicación. La omisión respecto a combatir la contaminación desde el ámbito legal (sea a través de la imposición de mayores exigencias legales como de los respectivos controles), también constituye en sí misma una falta importante y atenta contra el nivel de calidad del servicio.

La ley 13.036 sobre la comercialización del agua es una norma breve pero muy relevante en su contenido, ya que es la única sobre dicha temática que existe a nivel provincial, y que podría servir como antecedente para otros proyectos de leyes pertenecientes a otras jurisdicciones.
En su artículo 1 se prohíbe la comercialización del agua dulce o potable a granel y sin tratamiento o proceso de ningún tipo, obtenida de fuentes agotables superficiales o subterráneas, del dominio originario de la provincia, que implique directa o indirectamente su exportación, con las excepciones autorizadas en la presente ley: " 1 . Razones humanitarias o de emergencia. 2. Ejecución de convenios suscriptos con la Nación, con otras provincias, regiones, Estados u organismos internacionales".

El trámite para quedar encuadrado en el artículo 1 deberá contener, como mínimo y sin perjuicio de los demás recaudos que se establecerán por vía reglamentaria, la expresión del volumen, estado y demás características del agua que se intenta comercializar, los motivos por los cuales se pretende hacerlo, el destino, la identificación de la fuente de la cual se extraerá, los medios que se utilizarán para la extracción y la acreditación de la inexistencia o inocuidad de perjuicios para el ambiente, a través de un estudio y evaluación de impacto ambiental.

Evaluadas estas condiciones por la autoridad de aplicación y si se considerara viable la pretensión, se elevará la solicitud al poder 
ejecutivo para su autorización y se remitirán los antecedentes a la legislatura, para su conocimiento. Se imponen sanciones ante la violación a las disposiciones de la presente ley, con multas administrativas que serán graduadas y aplicadas por la autoridad de aplicación, cuyos montos están desactualizados y son bajos.

Quien haya sido sancionado por aplicación de esta ley y reincidiera en nueva violación en el término de cinco años contados desde la anterior sanción, será pasible de la aplicación de la máxima graduación de la multa. El producido de las mismas y del canon que establece el artículo $6^{\circ}$ de la presente ley se destinará a estudios, proyectos y ejecución de obras de extensión de redes de agua potable y saneamiento.

Además, no existen sanciones penales ni civiles significativas que tiendan a lograr un cambio de conducta en la ciudadanía. Sin embargo cabe destacar que, en el caso de esta norma se excedió lo establecido por el principio de subsidiariedad ${ }^{9}$ y por el artículo 41 de la Constitución Nacional, ya que en el caso de la provincia de Santa Fe se ha superado ampliamente al contenido de la norma nacional de presupuestos mínimos ${ }^{10}$, más allá de que la misma no pertenezca a esta última categoría.
La provincia de Santa Fe posee un desarrollo normativo ambiental muy avanzado en comparación con otras jurisdicciones, en particular porque prohíbe la comercialización del agua que implique su exportación. El artículo 2 de la norma dispone también en su inciso a), que una de sus finalidades es "garantizar el mantenimiento y propender a la rehabilitación, mejora y desarrollo del servicio en todo el ámbito de la provincia de Santa Fe".

Estas dos normas jurídicas provinciales que fueron analizadas pueden identificarse claramente con los postulados de los paradigmas de protección ambiental y de la administración de los recursos, porque se proponen metas prácticas y técnicas a los fines de suministrar agua potable, sin desplegar conceptos vinculados con la preservación de los ecosistemas donde se encuentren ubicados los recursos hídricos, no tendiendo a alcanzar una eventual recomposición o superación en los niveles actuales de protección ambiental.

\section{El fundamental aporte del derecho ambiental internacional a la protec- ción del agua}

En la tabla 3 se destacan los principales instrumentos internacionales ambientales, y en la tabla 4 se hace referencia a los especializados en la temática del agua dulce.

Tabla 3

Instrumentos internacionales ambientales

\begin{tabular}{|lccc|}
\hline \multicolumn{1}{|c|}{ Fuente legal } & $\begin{array}{c}\text { Enfoque } \\
\text { protecciónambiental }\end{array}$ & $\begin{array}{c}\text { Enfoque administración } \\
\text { de los recursos }\end{array}$ & $\begin{array}{c}\text { Enfoque } \\
\text { ecodesarrollo }\end{array}$ \\
\hline Declaración de Estocolmo (1972) & $\mathrm{X}$ & $\mathrm{X}$ \\
\hline Carta Mundial de la Naturaleza (1982) & $\mathrm{X}$ & $\mathrm{X}$ \\
\hline $\begin{array}{l}\text { Declaración de Río de Janeiro sobre el } \\
\text { medio ambiente y el desarrollo (1992) }\end{array}$ & $\mathrm{X}$ & $\mathrm{X}$ & \\
\hline Agenda XXI (1992) & $\mathrm{X}$ & $\mathrm{X}$ & \\
\hline Declaración del Milenio (2000) & $\mathrm{X}$ & $\mathrm{X}$ & \\
\hline Declaración de Malmö (2000) & $\mathrm{X}$ & $\mathrm{X}$ & \\
\hline $\begin{array}{l}\text { Objetivos de Desarrollo del Milenio } \\
\text { (2000-2015) }\end{array}$ & $\mathrm{X}$ & & \\
\hline
\end{tabular}




\begin{tabular}{|c|c|c|c|}
\hline Fuente legal & $\begin{array}{c}\text { Enfoque } \\
\text { protecciónambiental }\end{array}$ & $\begin{array}{c}\text { Enfoque administración } \\
\text { de los recursos }\end{array}$ & $\begin{array}{c}\text { Enfoque } \\
\text { ecodesarrollo }\end{array}$ \\
\hline $\begin{array}{l}\text { Declaración de Johannesburgo sobre el } \\
\text { desarrollo sostenible (2002) }\end{array}$ & $\mathrm{X}$ & $\mathrm{X}$ & \\
\hline $\begin{array}{l}\text { Objetivos de Desarrollo Sostenible de la } \\
\text { ONU (2015-2030) }\end{array}$ & $\mathrm{X}$ & $\mathrm{X}$ & \\
\hline
\end{tabular}

Fuente: elaboración propia.

Tabla 4

Instrumentos internacionales sobre agua dulce

\begin{tabular}{|c|c|c|c|}
\hline Fuente legal & $\begin{array}{c}\text { Enfoque protección } \\
\text { ambiental }\end{array}$ & $\begin{array}{l}\text { Enfoque administración } \\
\text { de los recursos }\end{array}$ & $\begin{array}{c}\text { Enfoque } \\
\text { ecodesarrollo }\end{array}$ \\
\hline $\begin{array}{l}\text { Carta Europea del Agua de Estrasburgo } \\
\text { (1968) }\end{array}$ & $X$ & $X$ & \\
\hline $\begin{array}{l}\text { Convención de la ONU sobre el derecho } \\
\text { de los usos de los cursos de agua } \\
\text { internacionales para fines distintos a la } \\
\text { navegación (1997) }\end{array}$ & $X$ & $X$ & \\
\hline $\begin{array}{l}\text { Resolución 64/292 de la Asamblea General } \\
\text { de la ONU (2010) }\end{array}$ & $X$ & $X$ & \\
\hline $\begin{array}{l}\text { Resolución } 68 / 157 \text { de la Asamblea General } \\
\text { de la ONU (2013) }\end{array}$ & $X$ & $X$ & \\
\hline $\begin{array}{l}\text { Resolución A/HRC/RES/27/7 del Consejo } \\
\text { de Derechos Humanos de la ONU (2014) }\end{array}$ & $X$ & $X$ & \\
\hline
\end{tabular}

Fuente: elaboración propia.

En el ámbito jurídico mundial puede considerarse que las conferencias de Estocolmo de 1972, la de Nueva York de 1982 (donde se firmó la Carta Mundial de la Naturaleza) y la de Río de Janeiro de 1992, a través de sus declaraciones constituyeron los primeros avances que generaron la interrelación del derecho internacional con el ambiente.

Luego, la Declaración del Milenio del año 2000 y la Declaración de Johannesburgo del 2002 también dictadas por la Asamblea General de las Naciones Unidas, fueron consecuencia histórica y evolutiva de diversos documentos que trataron los temas de la pobreza y del hambre mundial, y que principalmente derivan de los propósitos y principios de la Carta de las Naciones Unidas.

La Declaración de la Conferencia de las Naciones Unidas sobre el Medio Ambiente
Humano firmada en Estocolmo en 1972, manifiesta que todos los seres humanos tienen la obligación de proteger los recursos naturales, incluyendo específicamente el agua, para el uso de las generaciones presentes y futuras. Otro tema importante que se analizó en esta conferencia, fue la exigencia dirigida a todos los Estados para que afiancen sus políticas o sea que se empiece a tomar más conciencia sobre la gravedad del tema ambiental.

Por otro lado, se le reconoce a los Estados el derecho soberano de explotar sus recursos naturales, pero al mismo tiempo asegurar que las actividades que desarrollen no perjudiquen al ambiente.

En 1982 se aprobó la Carta Mundial de la Naturaleza por parte de la Asamblea General de las Naciones Unidas, donde se establecieron 
principios generales y fundamentales. Para empezar, se propugnó el respeto a la naturaleza y a sus procesos esenciales, se exigió que no se utilicen los recursos biológicos más allá de su capacidad natural de regeneración, y que los recursos no renovables y fungibles se deberán explotar de forma razonable. Con relación al agua, se expresó que se deberá reaprovechar o reciclar luego de su uso. Así mismo, al igual que otros documentos internacionales, se solicitó que los principios de dicha Carta se incorporen en el derecho local y en la práctica de cada Estado.

En 1992 se firmó la Declaración de Río sobre el Medio Ambiente y Desarrollo. En este documento se repitió la exigencia de que los Estados promulguen leyes eficaces para la protección del ambiente. Por otra parte, se fijan diversos principios como el de instituir una relación armónica entre la paz, el desarrollo y la protección del ambiente, la necesidad de que exista consenso entre los Estados para cuestionar actividades contaminantes realizadas por alguno de ellos, y el respeto a la buena fe. El Principio 15 de dicha declaración denominado "Precautorio", determina que

[...] con el fin de proteger el medio ambiente, los Estados deberán aplicar ampliamente el criterio de precaución conforme a sus capacidades. Cuando haya peligro de daño grave o irreversible, la falta de certeza científica absoluta no deberá utilizarse como razón para postergar la adopción de medidas eficaces en función de los costos para impedir la degradación del medio ambiente.

Varios autores (Curry, 2006, p. 48) sostienen que es necesario que se diseñen programas ecológicos que incluyan un principio precautorio fuerte, que implique que nuestro accionar debe ser cauteloso, pues se considera que el conocimiento de sus efectos siempre es inferior a nuestra ignorancia. De esta Cumbre de Río surgió uno de los instrumentos internacionales de mayor importancia, que marcó un precedente en numerosas agendas locales y que se denominó Agenda XXI.
Esta puede definirse como un programa político que se focaliza en la aplicación del principio de desarrollo sostenible. La característica primordial de este documento internacional, es que no persigue una finalidad teórica y que su contenido es netamente práctico y puede ser aplicado y adaptado en cualquier lugar del mundo.

En la Agenda XXI se repiten los objetivos tendientes a lograr una eficiente cooperación internacional y una reducción de la pobreza, teniendo en cuenta los tres pilares contenidos en el principio del desarrollo sostenible (económico, social y ambiental).

Así mismo, la Convención de las Naciones Unidas de 1997 sobre el derecho de los usos de los cursos de agua internacionales para fines distintos a la navegación, establece principios muy importantes, que ya se venían aplicando consuetudinariamente.

En primer lugar, la obligación de no causar un perjuicio apreciable de un Estado a otro, con la utilización de un curso de agua, en cuanto a su caudal o calidad. En segundo lugar, todos los países deben hacer un uso equitativo y razonable de las aguas, para que no se perjudiquen entre ellos. También se consagra la obligación de comunicar a otros Estados los proyectos de obras o trabajos hidráulicos que se realizarán en un futuro próximo.

Para analizar este aspecto, es importante comenzar con la consideración de uno de los principios fundamentales del derecho ambiental internacional, que es el de la "prevención del daño ambiental transfronterizo". El principio 21 de la Declaración de Estocolmo lo describe de la siguiente manera: "Los Estados tienen la obligación de asegurar que las actividades que se lleven a cabo dentro de su jurisdicción o bajo su control no perjudiquen al medio de otros Estados o de zonas situadas fuera de toda jurisdicción nacional".

Luego, en la Cumbre Mundial de la tierra celebrada en Johannesburgo en el 
año 2002, los principales temas fueron: la pobreza, la energía, el calentamiento global, los recursos naturales y la biodiversidad. Una de las propuestas planteadas que más se vincula con el presente trabajo, fue la de reducir a la mitad el número de personas que viven sin agua corriente y sin acceso a servicios sanitarios para el año 2015. Esto también se había propuesto a través de la implementación de metas concretas registradas en los Objetivos de Desarrollo del Milenio (con vigencia en los años 2000-2015). En este caso el número 7 proponía garantizar la sostenibilidad del medioambiente y "reducir a la mitad, para 2015, la proporción de personas sin acceso sostenible al agua potable".

Dentro de este se fijó un indicador (7.7) que planteaba la necesidad de "proporcionar a la población acceso a mejores fuentes de agua potable"11. Lo relevante de este aspecto es que la preocupación por el agua potable es significativa en todo el mundo, porque la carencia de este recurso está íntimamente relacionada con la salud humana, y con la imposibilidad de poder elevar el nivel de vida de las personas.

Por otro lado, uno de los más antiguos documentos internacionales vinculados con este recurso natural, es la Carta Europea del Agua firmada en Estrasburgo en 1968. Allí se hizo mención a los postulados más relevantes sobre el agua, en donde se intenta conseguir que los Estados conozcan que el inadecuado uso del líquido perjudica a los ecosistemas y a sus componentes, y también menciona la necesidad de legislar en el ámbito internacional sobre la protección de este recurso. Otro elemento fundamental que surge de la lectura de la Carta, es que es de vital importancia que se investigue sobre el agua y que se fomente la formación de profesionales especializados.

Tanto la Declaración del Milenio como la de Johannesburgo dieron lugar a la inminente aparición de otros documentos que profundizaron sobre aspectos que analizaron los primeros. Tal es el caso de la Declaración de Malmö del 2000, donde específicamente los países participantes establecieron que debe lograrse un consumo sostenible, en especial en los países más desarrollados. Así mismo, que la degradación ambiental y la pobreza están directamente relacionadas con el deterioro de los recursos naturales.

El hecho de que muchos ciudadanos no gocen de derechos humanos básicos, inhabilita a estos para que puedan desplegar sus capacidades individuales. Otro aspecto cardinal que ha sido planteado directa o indirectamente en ambos documentos, es considerar que los derechos a una alimentación adecuada y a vivir en condiciones dignas constituyen derechos humanos innegables para todas las personas. Esta postura brinda más firmeza y fuerza a las metas que se señalaron en los años 2000 y 2002 (del Milenio y Johannesburgo).

En concreto, en Johannesburgo se sostuvo que se debe promover la integración del crecimiento económico, del desarrollo social y de la protección del ambiente de manera general, incluyendo a todos los recursos naturales. En dicha Cumbre se fijaron objetivos de carácter más operativo y no tan programáticos o teóricos como en la Cumbre de Estocolmo de 1972.

Además, en la Declaración del Milenio se incorporó la necesidad de defender los principios de la dignidad humana y la igualdad mundial, los cuales se relacionan directamente con la problemática planteada aquí. Respecto al mismo contexto, el 28 de julio de 2010 en el $64^{\circ}$ periodo de sesiones, en el tema 48 del programa "Aplicación y seguimiento integrados y coordinados de los resultados de las grandes conferencias y cumbres de las Naciones Unidas en las esferas económica y social y esferas conexas", la Asamblea General de las Naciones Unidas adoptó una resolución muy relevante.

El 30 de septiembre de 2010, el Consejo de Derechos Humanos de las Naciones Unidas 
reconoció y ratificó que el acceso al agua potable y al saneamiento por parte de cualquier persona constituye un derecho humano básico para la vida; se le exigió a todos los Estados y a las organizaciones internacionales que aporten recursos financieros y que fomenten el aumento de la capacidad y de la transferencia de la tecnología, mediante la asistencia y la cooperación internacional (Minaverry \& Martínez, 2015, p. 2).

Lo anterior implicó un claro reconocimiento de un derecho humano que compromete a todos los Estados a garantizar a sus habitantes la provisión del servicio de agua potable y de saneamiento, lo que conlleva asumir los costos que impliquen los servicios. En la misma no se estableció la manera ni los medios que deben utilizarse para conseguir esto, por lo que cada Estado posee libertad de acción en este sentido.

Posteriormente, la resolución $\mathrm{A} / \mathrm{HRC} /$ RES/27/7 la dictó el Consejo de Derechos Humanos en septiembre de 2014, en este instrumento se enumeran las fuentes respecto de las cuales surge el reconocimiento del derecho humano.

En este sentido menciona, en primer lugar, a la resolución 64/292 de la Asamblea General, de 28 de julio de 2010, en la que la Asamblea reconoció que el derecho al agua potable y al saneamiento es un derecho humano esencial para el pleno disfrute de la vida y de todos los derechos humanos, así como la resolución 68/157 del 18 de diciembre de 2013, en la que la Asamblea reafirmó por consenso el derecho humano al agua potable y al saneamiento (Minaverry \& Martínez, 2015, p. 7).

La resolución en análisis destacó que las cifras oficiales no reflejan plenamente las dimensiones de la salubridad del agua potable y la asequibilidad de los servicios, y subestiman el número de personas sin acceso a agua potable salubre. Además, resalta en este contexto la necesidad de vigilar adecuadamente la calidad del agua potable y la seguridad del saneamiento, a fin de obtener datos que evidencien esas dimensiones como algo fundamental para garantizar el acceso al agua potable.

Más recientemente, los Objetivos de Desarrollo Sostenible de las Naciones Unidas (2015-2030) hicieron un aporte esencial en relación con la protección del agua y del ambiente en general. El objetivo número 6 determina que se debe "garantizar la disponibilidad de agua y su gestión sostenible y el saneamiento para todos". Allí se sugieren puntualmente metas cuantificables y medibles, con el propósito de lograr avances concretos respecto del acceso a una excelente calidad y disponibilidad del agua:

- Para 2030, lograr el acceso universal y equitativo al agua potable, a un precio asequible para todos.

- Para 2030, mejorar la calidad del agua mediante la reducción de la contaminación, la eliminación del vertimiento y la reducción al mínimo de la descarga de materiales y productos químicos peligrosos, la reducción a la mitad del porcentaje de aguas residuales sin tratar y un aumento sustancial del reciclado y la reutilización en condiciones de seguridad a nivel mundial.

- Para 2030, aumentar sustancialmente la utilización eficiente de los recursos hídricos en todos los sectores y asegurar la sostenibilidad de la extracción y el abastecimiento de agua dulce para hacer frente a la escasez de agua y reducir sustancialmente el número de personas que sufren de escasez de agua.

- Para 2030, poner en práctica la gestión integrada de los recursos hídricos a todos los niveles, incluso mediante la cooperación transfronteriza, según proceda.

- Para 2020, proteger y restablecer los ecosistemas relacionados con el agua, incluidos los bosques, las montañas, los 
humedales, los ríos, los acuíferos y los lagos.

- Para 2030, ampliar la cooperación internacional y el apoyo prestado a los países en desarrollo para la creación de capacidad en actividades y programas relativos al agua y el saneamiento, incluidos el acopio y almacenamiento de agua, la desalinización, el aprovechamiento eficiente de los recursos hídricos, el tratamiento de aguas residuales y las tecnologías de reciclaje y reutilización.

- Apoyar y fortalecer la participación de las comunidades locales en la mejora de la gestión del agua y el saneamiento.

Respecto del documento "Transformando nuestro mundo la Agenda 2030 para el Desarrollo Sostenible" se realizó un seguimiento de los resultados de la Cumbre del Milenio (planteados para el periodo 2000-2015). Allí se hace referencia a la situación actual de nuestro mundo, y se establece que el desarrollo sostenible afronta inmensos desafíos, debido al crecimiento de las desigualdades.

Lo anterior se refleja claramente en la reducción de las condiciones de vida y sus impactos en la salud de las personas, en la frecuencia y en la intensidad de los desastres naturales, en la escalada de los conflictos bélicos, en el extremismo violento, el terrorismo $\mathrm{y}$ en la aparición de crisis humanitarias y desplazamientos migratorios.

Además debe tenerse en cuenta que recientemente finalizó el Decenio Internacional para la Acción "El agua fuente de vida 20052015", donde se reconoció que a lo largo del mismo se trabajó conjuntamente para lograr avances en la concreción de los objetivos, que se había propuesto la comunidad internacional en relación con alcanzar un mejor acceso al agua y al saneamiento.

Además se hizo foco en los programas y proyectos ambientales que lleva adelante cada
Estado, y que previamente habían sido adaptados a un contexto local particular, para conseguir que se implementen avances respecto a los aspectos analizados en el presente artículo ${ }^{12}$.

\section{Algunas recomendaciones}

En el ámbito internacional ambiental, deben considerarse propuestas asociadas a la creación de un tribunal especializado en la protección del ambiente, para poder intentar cuantificar legalmente el valor de los bienes ambientales de los ecosistemas. La misma problemática debe resolverse en el ámbito local, en tanto que

[...] una de las cuestiones centrales vinculadas con la importancia de la existencia de estos organismos es que las cortes y tribunales ambientales pueden estar facultados para adoptar enfoques integrados que permitan abarcar las diferentes leyes ambientales en su conjunto, a diferencia de lo que ocurre con los tribunales ordinarios que no se encuentran capacitados para actuar de esta forma (Minaverry, 2015, p. 106).

De esta manera se podrá proteger mejor a los mismos en el ámbito internacional, y que no se dicten resoluciones ni sentencias que no interpreten adecuadamente las cuestiones ambientales más técnicas (Minaverry, 2016). Luego, del análisis exhaustivo de los instrumentos internacionales que sientan principios generales aplicables al ámbito del derecho ambiental y de los sectoriales que protegen únicamente al agua, se puede concluir que el recurso se encuentra jurídicamente protegido en un sentido amplio. Sin embargo, es evidente que escasean instrumentos específicos vinculantes sobre gestión y manejo del agua en conjunto con sus ecosistemas.

Es conveniente sugerir que se comience a redactar un protocolo estableciendo obligaciones para todos los Estados, respecto al uso del agua, para que cada país pueda tener control y proteger sus recursos de agua dulce de forma 
inmediata, pero lo más importante es que la investigación y la elaboración de documentos internacionales continúen, y permitan un avance favorable sobre el tema en un futuro próximo.

En el ámbito de la normativa ambiental nacional, en términos generales, no se han aplicado enfoques ecosistémicos que tomen en consideración el todo (en este caso los ecosistemas del agua), mientras que se han detectado los unisistémicos que focalizan la atención en un componente particular del ecosistema (Capaldo, 2009). En nuestro contexto (salvo algunas excepciones), las normas en la actualidad protegen legalmente a los diferentes recursos por separado y no de manera integral.

Por tal razón, sería recomendable seguir en el futuro los criterios plasmados, por ejemplo, por la normativa nacional sobre bosques nativos, en donde se lo define como

$[\ldots]$ los ecosistemas forestales naturales compuestos predominantemente por especies arbóreas nativas maduras, con diversas especies de flora y fauna asociadas, en conjunto con el medio que las rodea -suelo, subsuelo, atmósfera, clima, recursos hídricos-, conformando una trama interdependiente con características propias y múltiples funciones, que en su estado natural le otorgan al sistema una condición de equilibrio dinámico y que brinda diversos servicios ambientales a la sociedad, además de los diversos recursos naturales con posibilidad de utilización económica (Ley nacional 26.331 de 2007, art. 2).

En este sentido podemos vislumbrar la necesidad de que la implementación de las normas jurídicas ambientales se realice dentro de un marco de interdisciplinariedad, donde las diferentes ciencias puedan brindar sus herramientas (derecho, geografía, economía, sociología, entre otras), ya que de ese modo podrá regularse más adecuadamente la relación existente entre la naturaleza y la ciudadanía (Minaverry, 2016, p. 26).
Esto es así porque "los científicos ambientales no son ciegos a que el humano es un producto de la complejidad ecosistémica [...] y por lo tanto estas ciencias conciben relaciones recursivas entre la naturaleza no antroposocial y las antroposociedades [...]" (Morales-Jasso, 2016 , p. 143), y sería muy importante que estos enfoques se trasladen a las normas jurídicas ambientales, para que su efectividad sea mayor.

El derecho ambiental hoy en día ofrece herramientas adecuadas y relevantes para lograr una mayor protección integral del ambiente, y este caso es muy claro cuando nos referimos al procedimiento administrativo de las evaluaciones de impacto ambiental, donde "legalmente" se cumplimenta con todos los requisitos legales vigentes, a pesar de que con frecuencia no se observan muchos efectos positivos en la práctica.

Una posible opción para intentar subsanar esto sería "recurrir a evaluaciones multicriteriales participativas donde se intente que tanto las alternativas en cuestión como los criterios o valores a tener en cuenta, salieran de una amplia discusión entre empresas, gobiernos, los afectados localmente y otros interesados" (Martínez-Alier, 2015, p. 67).

Los aspectos sociales deben ser decididamente considerados al momento de sancionar normas jurídicas, como ocurre en el caso del ecologismo popular, donde son "una expresión de una serie de estrategias de resistencia al poder que enfrenta a la economía de mercado representada por los grupos de poder económico y político, con la economía moral de los pobres marginados de la distribución ecológica" (Goebel-Mc Dermott, 2015, p. 131). Sin duda las mismas surgieron como consecuencia de la excesiva lentitud de los representantes del Estado que deberían defender los derechos colectivos, como es el caso de los ambientales.

El ambiente implica las interacciones entre sociedad y naturaleza -lo que incluye a 
las sociedades no antrópicas-, es algo que no se puede remplazar con ningún sustituto tecnológico y su valor económico es incalculable (MoralesJasso, 2016, p. 150). El empoderamiento de la sociedad civil, que cuenta con herramientas tecnológicas de información y de comunicación, le ha permitido tomar y manifestar su posición frente a las prácticas ambiental y socialmente inadecuadas en todo el mundo (GuzmánAguilera, 2013, p. 19).

Por su parte, la tendencia que sigue la normativa nacional argentina es opuesta, y esto se nota en el texto de la ley nacional $25.688 \mathrm{de}$ presupuestos mínimos para la gestión ambiental de agua de 2002. En su artículo 6 establece que para la utilización de los recursos hídricos, debe contarse con un permiso otorgado por la autoridad competente. En su artículo 7 afirma que dicha autoridad deberá:

a) Determinar los límites máximos de contaminación aceptables para las aguas de acuerdo a los distintos usos;

b) Definir las directrices para la recarga y protección de los acuíferos;

c) Fijar los parámetros y estándares ambientales de calidad de las aguas;

d) Elaborar y actualizar el Plan Nacional para la preservación, aprovechamiento $\mathrm{y}$ uso racional de las aguas.

Esta norma no detalla mecanismos, límites específicos ni una correcta descripción respecto de un tema que posee tanta trascendencia, ya que aún no ha sido reglamentada por parte del Poder Ejecutivo Nacional, pero muchos reglamentos la invocan, y la citan algunos fallos desde el momento de su sanción (Valls, 2012, p. 268). Al no contar con una norma nacional que regule y otorgue principios básicos para que las provincias argentinas puedan complementarlos a través del dictado de leyes provinciales, se recomienda en el futuro tomar en cuenta varios de los proyectos de leyes que incluyeron dichos aspectos, que tramitan actualmente ante el Congreso Nacional, y también otros que ya perdieron estado parlamentario.

Debe considerarse el texto de la ley nacional 26.639 de presupuestos mínimos para la preservación de los glaciares y del ambiente periglacial de 2010, en su artículo 1 ubica en el mismo nivel de jerarquía al uso del agua para consumo humano, como atractivo turístico y para la agricultura.

El texto de la ley nacional 26.418 de presupuestos mínimos para la preservación de los glaciares y del ambiente periglacial de 2008 (que vetó el Poder Ejecutivo Nacional mediante el decreto 1837 de 2008 sobre política ambiental nacional), no había incluido como finalidad de protección a los recursos hídricos con destino a actividades agrícolas. En este último caso, la prioridad respecto de la protección del recurso se enfocaba únicamente hacia el consumo humano, y para su protección como reservorios.

\section{Conclusiones}

Podemos concluir que ambos ordenamientos jurídicos provinciales (Buenos Aires y Santa $\mathrm{Fe}$ ) poseen un fuerte enfoque antropocéntrico, al privilegiar los beneficios del hombre por sobre los derechos de la naturaleza/ambiente, y no profundizar en otros aspectos fundamentales como son los sociales, ambientales y culturales.

Prácticamente ninguna de las dos normativas reúne características compatibles con el enfoque del ecodesarrollo de Colby, pero ambas reconocen en sus textos derechos humanos de tercera generación (ya que el Estado asume obligaciones concretas), en tanto que incorporan a la protección del ambiente pero en función de los derechos de las personas y no tendientes a lograr la integridad de los ecosistemas.

Se ha detectado un seguimiento de los esquemas más tradicionales donde el manejo del ambiente se estructura principalmente 
para lograr metas prácticas, desconociendo la visión ecosistémica que interrelaciona al agua $\mathrm{y}$ al ambiente, y que sugiere tratarse como un patrimonio de todos a preservar, más que como un flujo de beneficios por utilizar (Pérez, RojasPadilla, \& Galvis, 2013, p. 17).

Se debe tener en cuenta que en Argentina el marco regulatorio del agua es complejo, pues las competencias son concurrentes entre la nación y las provincias, y además se trata de un recurso ambiental fundamental para la vida.

La ley 25.688 que se analizó aquí, que establece los presupuestos mínimos ambientales sobre la gestión del agua, posee algunos enfoques que son inconsistentes, por lo que no brinda una guía recomendable a seguir por parte de las provincias, más allá de no haber sido reglamentada y de que no haya incorporado ninguno de los conceptos postulados en el paradigma de ecodesarrollo, que representa la tendencia hacia donde se dirige la mayor parte de la normativa ambiental de la región y de otros continentes.

Los cuestionamientos respecto de esta norma se orientan hacia las autoridades hídricas, puesto que las provincias efectuaron una presentación ante el subsecretario de Recursos Hídricos de la Nación solicitando su veto por parte del Poder Ejecutivo Nacional. En la misma alegaron la inconstitucionalidad de la ley por las siguientes razones:

- Haberse excedido la competencia delegada en el artículo 41 de la Constitución Nacional.

- La violación del deslinde de competencias.

- Haberse avanzado sobre aspectos no delegados a la nación y reservados a las jurisdicciones locales, como es la gestión de los recursos naturales (vulnerándose el artículo 124 de la Constitución Nacional ${ }^{13}$ ).
Por eso, más allá de que en este trabajo analizamos principalmente la normativa sobre aguas, podemos destacar que otras normas jurídicas ambientales ya se encuentran incorporando diversos principios, que derivan de contextos sociales diferentes al nacional y en donde se entrelazan más con estos movimientos regionales descriptos anteriormente, contemplando los aportes hechos por la participación ciudadana.

Sin duda, el principio de desarrollo sostenible se encuentra receptado en toda la normativa estudiada en estas jurisdicciones, pero su crítica se basa principalmente en que se limita a reorganizar el criterio de utilización económica de explotación de los recursos naturales, sin considerar la totalidad de los impactos negativos que se producen en el ambiente.

Todas las legislaciones deberían tener en cuenta esto, conforme se destaca en el "Manifiesto por la vida. Por una ética para la sustentabilidad", donde el concepto de sustentabilidad se funda en el reconocimiento de los límites y potenciales de la naturaleza.

Estos aspectos en general no se ven receptados en los textos normativos estudiados aquí. El enfoque es más sectorial y al especializarse tanto en la temática del agua, no considera que a corto, mediano o largo plazo, según sea el caso, esto va a repercutir negativamente en el ambiente y en la naturaleza $\mathrm{y}$ acto seguido en la misma población; ni mucho menos considera el líquido como un derecho humano. De hecho, dicha categoría fue eliminada del anteproyecto de Código Civil y Comercial de la Nación de Argentina que fue sancionado en $2015^{14}$.

Analizando el asunto únicamente desde el ámbito económico, también podríamos destacar que los costos de no proteger al ambiente de manera holística, se reflejan directamente en el incremento de gastos que deberán afrontar los Gobiernos a través de los impuestos que 
pagan todos los ciudadanos, para recomponer el ambiente, indemnizar a particulares, entre otras cuestiones, tal como aparece reflejado en el conocido Informe Stern.

Del examen de los instrumentos internacionales podemos destacar que los vinculados con la protección del ambiente en general, poseen un enfoque que está más orientado hacia el paradigma del ecodesarrollo, en especial los que se encuentran cronológicamente ubicados en los años 1972 y 1982, y en 2015 a través de los Objetivos de Desarrollo Sostenible (20152030). Estos últimos incluyen mayor cantidad de objetivos que consideran a los ecosistemas como sistemas en sí mismos, protegiendo a todos sus elementos constituyentes (número 15: sobre la vida y los ecosistemas; número 14: sobre la vida submarina y número 6: sobre agua y saneamiento).

En cambio, los instrumentos internacionales especializados en la protección del agua tienen una orientación más proclive a la utilización práctica del recurso, sin tener tanto en cuenta sus consecuencias ambientales, ya que plantean aspectos asociados con la implementación de planes, programas y actividades concretas que están más asociados con ofrecer un acceso rápido al agua a la máxima cantidad de personas posible.

Esto es comprensible y se condice con lo establecido al comienzo del presente artículo, cuando se hacía referencia a la crisis del agua y a su importancia en relación con su incorporación en las agendas políticas, lo cual se conecta con el dictado de las normas jurídicas y de las herramientas internacionales ambientales. Sin embargo y afortunadamente, el derecho internacional ambiental se encuentra actualmente posicionando al derecho humano de acceso al agua, a través del contenido de sus diversos instrumentos.

Por último, al lograr visualizarse las valoraciones ambientales ya existentes en la norma- tiva vigente, las mismas podrían continuarse, fomentarse o defenderse a través de su aplicación y control por parte de los organismos ambientales responsables, y servir como posible guía de lineamientos para futuros aportes realizados desde el campo del derecho.

Si la regulación vigente comienza a tomar en cuenta ciertos aspectos que de alguna manera exceden lo estrictamente jurídico, será posible que se eviten o mitiguen ciertos fenómenos sociales que son perjudiciales y mejorar la dignidad y calidad de vida de las poblaciones.

\section{Notas}

1 Aprueba el marco regulatorio para la prestación de los servicios públicos de provisión de agua potable y desagües cloacales en la Provincia de Buenos Aires.

${ }^{2}$ Código de Aguas de la Provincia de Buenos Aires de 1999.

${ }^{3}$ Hace referencia al derecho de acceso al agua potable y al saneamiento como derecho humano esencial para la vida. Fue sancionada el 16 de septiembre de 2015 y publicada el 21 de diciembre del mismo año.

4 Aprueba el marco regulatorio para la prestación de los servicios públicos de provisión de agua potable y desagües cloacales en la Provincia de Buenos Aires.

${ }^{5}$ Para mayor información véase: Cáceres, 15 de febrero de 2015.

${ }^{6}$ Data del año 1962, en donde la temática ambiental no era regulada por prácticamente ningún texto normativo.

7 Sobre la transformación del sector público del agua potable, desagües cloacales y saneamiento.

8 Sobre la comercialización del agua. 
9 El Estado a través de las distintas instancias de la administración pública, tiene la obligación de colaborar $\mathrm{y}$, de ser necesario, participar en forma complementaria en el accionar de los particulares en la preservación y protección ambientales.

${ }^{10}$ La ley 25.688 es amplia y en la misma no aparecen planteados diversos aspectos que han sido tratados detalladamente por la normativa de la Provincia de Santa Fe.

${ }^{11}$ Para más información sobre este tema, véase: http://www.un.org/es/millenniumgoals/ pdf/2015/mdg-report-2015_spanish.pdf

12 Para más información véase: http:// www.un.org/spanish/waterforlifedecade

${ }^{13}$ Las provincias podrán crear regiones para el desarrollo económico y social y establecer órganos con facultades para el cumplimiento de sus fines; podrán también celebrar convenios internacionales en tanto no sean incompatibles con la política exterior de la nación y no afecten las facultades delegadas al Gobierno federal o el crédito público de la nación; con conocimiento del Congreso Nacional. Buenos Aires tendrá el régimen que se establezca a tal efecto. Corresponde a las provincias el dominio originario de los recursos naturales existentes en su territorio.

14 Para mayor información véase Minaverry y Martínez (2016).

\section{Referencias}

Cáceres, V. (15 de febrero de 2015). La situación de ABSA en la provincia de buenos aires. Página/12. Recuperado de http://www. pagina12.com.ar/diario/suplementos/cash/ 17-8295-2015-02-15.html

Capaldo, G. (2009). El rol de los ecosistemas en la ecuación del agua. Jornadas Interdisciplinarias de Derecho Ambiental, Mendoza, Argentina.
Carta Europea del agua (1968). Estrasburgo. Recuperado de http://tragua.com/wpcontent/uploads/2012/04/Carta_Europea del_Agua.pdf

Colby, M. (1991). La Administración en el Desarrollo: Evolución de los paradigmas. El Trimestre Económico, 58(231), 589-615.

Consejo de la Tierra. (2000). Carta de la Tierra. Holanda. Recuperado de http:// earthcharter.org/invent/images/uploads/ echarter_spanish.pdf

Curry, P. (2006). Ecological Ethics. Cambridge: Polity Books.

De Bianchetti, A. (s.f). El uso agrícola del agua. Argentina: Universidad Nacional del Nordeste.

De Bianchetti, A. (2010). Tarifa de agua potable y servicios ambientales. Análisis $y$ propuestas en el caso de Corrientes. Buenos Aires: Microjuris.

Galano, C., et al. (2002). Manifiesto por la vida. Por una Ética para la sustentabilidad. Ambiente \& Sociedade, 5(10), 1-14. Recuperado de http://www.redalyc.org/ articulo.oa?id=31713416012

Goebel-Mc Dermott, A. (2010). Ecologismo de los pobres y margilidad social: vehículos de complementariedad y puentes dialógicos. Revista Reflexiones, 89(1), 127-142.

Guzmán-Aguilera, P. (2013). Biodiversidad, derecho y negocios. Cuestiones jurídicas y prácticas en torno a la biodiversidad y su uso en los negocios. Bogotá: Editorial Universidad Externado de Colombia.

Martínez-Alier, J. (2015). Ecología política del extractivismo y justicia socio-ambiental. Revista Interdisciplina, 3(7), 57-73. 
Minaverry, C. (2015). El avance de la implementación de los tribunales ambientales en América Latina. Gestión y Ambiente, 18(2), 95-108. Recuperado de http:// www.revistas.unal.edu.co/index.php/gestion/article/viewFile/49367/54407

Minaverry, C. (2016). La valoración ambiental de los servicios ecosistémicos que brinda el agua en el marco normativo de América del Sur. Revista Catalana de Dret Ambiental, 7(1). Recuperado de http://forum.rcda.cat/index.php/rcda/ article/view/658/3256

Minaverry, C., \& Cáceres V. (2016). La problemática del arsénico en el servicio de agua en la Provincia de Buenos Aires, Argentina, Revista internacional de contaminación ambiental, 32(1), 69-76.

Minaverry, C., \& Martínez, A. (2015). El reconocimiento judicial del derecho humano de acceso al agua potable en Argentina. Reflexiones a raíz del dictado del fallo Kersich Juan Gabriel en la Corte Suprema de Justicia de la Nación, Erreius online, $1,1-24$.

Minaverry, C., \& Martínez,A. (2016). El derecho de acceso al agua para consumo humano en el nuevo código civil y comercial de la nación de argentina. Actualidad Jurídica Ambiental, 57. Recuperado de http://www. actualidadjuridicaambiental.com/wpcontent/uploads/2016/04/2016_05_02 Minaverry-Martinez-Agua-Argentina.pdf

Molina-Roa, J. (2014). Derechos de la naturaleza. Historia y tendencias actuales. Bogotá: Editorial Universidad Externado de Colombia.

Morales-Jasso, G. (2016). La apropiación de la naturaleza como recurso. Una mirada reflexiva. Gestión y Ambiente, 19(1), 141154. Recuperado de http://www.redalyc. org/pdf/1694/169446378009.pdf
Organización de Naciones Unidas. (1972). Declaración de la Conferencia de las Naciones Unidas sobre el Medio Ambiente Humano (Declaración de Estocolmo). Estocolmo. Recuperado de http://www2. medioambiente.gov.ar/acuerdos/convenciones/estocolmo/estoc_declar.htm

Organización de Naciones Unidas. (1982). Carta Mundial de la Naturaleza. Nueva York: Autor. Recuperado de http:// www.mbigua.org.ar/uploads/File/ CartaMundialNaturaleza.pdf

Organización de Naciones Unidas. (1992). Declaración de Río sobre el medio ambiente y desarrollo. Río de Janeiro. Recuperado de http://www2.medioambiente.gov.ar/acuerdos/convenciones/rio92/declaracion.htm

Organización de Naciones Unidas. (1992). Agenda XXI. Río de Janeiro. Recuperado de http://www2.medioambiente.gov.ar/ acuerdos/convenciones/rio92/agenda21/ ageindi.htm

Organización de Naciones Unidas. (1997). Convención sobre el derecho de los usos de los cursos de agua internacionales para fines distintos a la navegación. Recuperado de http://www.solidaritat. ub.edu/observatori/general/docugral/ N9777296.pdf

Organización de Naciones Unidas. (2000). Declaración del Milenio. Nueva York. Recuperado de http://www.un.org/spanish/ milenio/ares552.pdf

Organización de Naciones Unidas. (2000). Declaración de Malmo. Suecia. Recuperado de http://www.unep.org/malmo/ declaraci\%C3\%B3n_ministerial_de_ malm $\%$ C3\%B61.htm

Organización de Naciones Unidas. (2000). Objetivos de Desarrollo del Milenio. Recu- 
perado de http://www.who.int/topics/millennium_development_goals/about/es/

Organización de Naciones Unidas. (2002). Declaración de Johannesburgo sobre el desarrollo sostenible. Recuperado de http://www.culturalrights.net/descargas/ drets_culturals412.pdf

Organización de Naciones Unidas. (2015). Objetivos de Desarrollo Sostenible. Recuperado de http://www.un.org/sustainabledevelopment/es/objetivos-de-desarro1lo-sostenible/

Pastorino, L. (2014). De códigos y desafios jurídicos para enfrentar la crisis del agua. Buenos Aires: Ediciones Cooperativas.

Pérez, A., Rojas-Padilla, J., \& Galvis, R. (2013). Sociedad y servicios ecosistémicos. Perspectivas desde la minería, los megaproyectos y la educación ambiental. Cali: Universidad del Valle.

República de Argentina. Constitución Nacional de la República Argentina. (1994). Convención Nacional Constituyente.

República de Argentina. Decreto 878 de 2003. Por el cual se propicia la modificación del Marco Regulatorio para la Prestación de los Servicios Públicos de Agua Potable y Desagües Cloacales, en la Provincia de Buenos Aires. Gobernación de la Provincia de Buenos Aires, noviembre de 2003.

República Argentina. Decreto 3511 de 2007. Reglamentación del Código de aguas de la Provincia de Buenos Aires. Gobernación de la Provincia de Buenos Aires, 2007.

República Argentina. Decreto 1837 de 2008. Observase el Proyecto de Ley registrado bajo el $\mathrm{N}^{\mathrm{o}}$ 26.418. Boletín Oficial del 10/11/2008. Honorable Congreso de la Nación, octubre de 2008.
República de Argentina. Ley 11.220 de 1994. Transformación del sector público de agua potable, desagües cloacales y saneamiento. Boletín Oficial del 12/12/1994. Senado y Cámara de Diputados de la Provincia de Buenos Aires, diciembre de 1994.

República de Argentina. Ley 12.257 de 1999. Código de aguas. Boletín Oficial No. 23.756. Senado y Cámara de Diputados de la Provincia de Buenos Aires, enero de 1999.

República de Argentina. Ley 25.675 de 2002. Ley general del ambiente. Boletín Oficial del 26/11/2002. Senado y Cámara de Diputados de la Nación de Argentina, noviembre de 2002.

República de Argentina. Ley 25.688 de 2002. Régimen de gestión ambiental de aguas. Boletín Oficial del 03/01/2003. Senado y Cámara de Diputados de la Nación de Argentina, enero de 2003.

República de Argentina. Ley 13.230 de 2004. La Provincia de Buenos Aires adhiere al Código Alimentario Argentino. Boletín Oficial del 29/09/2004. Senado y Cámara de Diputados de la Provincia de Buenos Aires, septiembre de 2004.

República de Argentina. Ley nacional 26.331 de 2007. Presupuestos mínimos para la protección de los bosques nativos. Boletín Oficial No. 31.310 del 19/12/2007. Senado y Cámara de Diputados de la Nación de Argentina, diciembre de 2007.

República de Argentina. Ley 13.036 de 2009. Reglamentación sobre comercialización de agua dulce o potable. Boletín Oficial del 14/12/2009. Senado y Cámara de Diputados de la Nación de Argentina, diciembre de 2009.

República de Argentina. Ley 26.418 de 2008. Presupuestos mínimos para la preserva- 
ción de los glaciares y del ambiente periglacial. Boletín Oficial del 22/10/2008. Senado y Cámara de Diputados de la Nación de Argentina, octubre de 2008.

República de Argentina. Ley 26.639 de 2010. Presupuestos mínimos para la preservación de los glaciares y del ambiente periglacial. Boletín Oficial del 28/10/2010. Senado y Cámara de Diputados de la Nación de Argentina, diciembre de 2010.

República de Argentina. Ley 14.782 de 2015. Por la cual se reconoce el acceso al agua potable y al saneamiento como un derecho humano esencial para la vida. Boletín oficial del 21/12/2015. Senado y Cámara de Diputados de la Provincia de Buenos Aires, diciembre de 2015.

República de Argentina. Resolución 64/292 de 2010. El derecho humano al agua y el saneamiento. Asamble General de la Organización de Naciones Unidas, julio de 2010. Recuperado de http://www.un.org/ ga/search/view_doc.asp?symbol=A/ RES/64/292\&Lang=S
República de Argentina. Resolución 68/157 de 2013. El derecho humano al agua y el saneamiento. AsambleGeneral de la Organización de Naciones Unidas, diciembre de 2013. Recuperado de http:// www.un.org/es/comun/docs/?symbol=A/ RES/68/157

República de Argentina. Resolución A/HRC/ $\mathrm{RES} / 27 / 7$ de 2014. Derecho humano al agua potable y al saneamiento. Consejo de Derechos Humanos de la Organización de Naciones Unidas, septiembre de 2014. Recuperado de https://documents-dds-ny. un.org/doc/UNDOC/GEN/G14/177/87/

PDF/G1417787.pdf?OpenElement

Valls, M. (2012). Presupuestos mínimos ambientales. Buenos Aires: Editorial Astrea.

Viciano-Pastor, R., Martínez-Dalmau, R. (s.f.). ¿Se puede hablar de un nuevo constitucionalismo latinoamericano como corriente doctrinal sistematizada?. Recuperado de http://www.juridicas.unam.mx/ $\mathrm{wccl} /$ ponencias/13/245.pdf 
